- Case Report

\title{
A Patient with Neutropenia and Splenomegaly: A Case Report from Department of Family Medicine in Tertiary Hospital Center
}

\author{
Jaehee Cho ${ }^{1,2}$, Youhyun Song ${ }^{2}$, Jiyeon Lee', Daeun Lee' ${ }^{2}$ Yunsun $\mathrm{Go}^{2}$, Hee Cheol Kang ${ }^{2, *}$ \\ ${ }^{1}$ Yonsei University College of Medicine, Seoul, Korea \\ ${ }^{2}$ Department of Family Medicine, Severance Hospital, Yonsei University College of Medicine, Seoul, Korea
}

\begin{abstract}
Pancytopenia represents a unique challenge for primary care doctors and its etiological causes encompass various specialties, including hematology and rheumatology. Despite the existence of effective tests such as bone marrow biopsy and immunoassays to rule out the potential causes of pancytopenia, it is often difficult to pinpoint the exact diagnosis. In this case report, we have described such a 'gray zone' patient, who presented with pancytopenia, neutropenia, and splenomegaly, and was being treated for fungal pneumonia before being transferred to Severance Hospital (department of family medicine). As the patient had a 10-year history of multiple, long-term hospital admissions that were having a severely debilitating impact on the quality of life, we performed a partial splenic embolization as a potential cure for the symptoms. Although this induced acute blood count recovery, it failed to prevent eventual mortality from septic shock.
\end{abstract}

Keywords: Pancytopenia; Neutropenia; Hypersplenism; Embolization, Therapeutic

Received: October 19, 2019, Revised: January 16, 2020, Accepted: January 18, 2020

*Corresponding Author: Hee Cheol Kang https://orcid.org/0000-0002-0309-7448

Tel: +82-2-2228-2332, Fax: +82-2-362-2473, E-mail: kanghc@yuhs.ac 


\section{INTRODUCTION}

Pancytopenia is a condition that can prove to be a diagnostic challenge for primary care physicians. It can be caused by a wide variety of etiologies, namely bone marrow failure, marrow infiltrating lesions, autoimmune disorders, infections, and nutritional deficiencies, ${ }^{1)}$ and is usually managed by a hematologist or a rheumatologist, depending on the etiology. As the potential causes are directly related to various anomalies of the bone marrow, a bone marrow biopsy is one of the most critical investigations for managing a patient with pancytopenia. ${ }^{1,2)}$ In Korea, malignancy is a common diagnosis from bone marrow biopsies, but a substantial percentage of patients have unspecific findings. ${ }^{2)}$ Recent terminology describes this condition as idiopathic cytopenia of undetermined significance (ICUS). ${ }^{3)}$ However, little is reported regarding the epidemiology and clinical characteristics of this condition. Once autoimmune causes are also ruled out, the affected patients are in what is described as a clinical 'gray zone' where neither hematologic nor rheumatologic management alone can completely provide patients with comprehensive medical care. This adversely affects their quality of life as well as clinical status. This case report describes the clinical history and management of a 69-year-old man with longstanding pancytopenia along with splenomegaly and neutropenia, in the department of family medicine at a Korean tertiary hospital. Accordingly, we have explored the role of family medicine in the management of such 'gray zone' patients.

\section{CASE REPORT}

A 69-year-old man was referred to the department of family medicine at Severance Hospital for chronic weakness and recurrent admissions for untreated and undiagnosed longstanding pancytopenia, which had persisted for the last 10 years. The patient had severe splenomegaly and was being treated for fungal pneumonia (aspergilloma and Pneumocystis jirovecii pneumonia) before the referral. There was past medical history of right thyroidectomy (for thyroid cancer), posterior right coronary artery stenting (due to chronic stable angina pectoris), and paroxysmal atrial fibrillation. The repeated admissions were severely crippling the patient's quality of life. The current admission was for exacerbation of fungal pneumonia. Upon initial evaluation, the pa- tient was afebrile, with body temperature of $36.5^{\circ} \mathrm{C}$ and blood pressure of 99/66 mm Hg. Heart rate was 77 beats per minute and respiratory rate was 20 per minute. The patient showed poor nutritional status, weighing $48.7 \mathrm{~kg}$ with a height of $169 \mathrm{~cm}$ (body mass index [BMI], $17.05 \mathrm{~kg} / \mathrm{m}^{2}$ ). The patient appeared cachectic and exhausted by prolonged admission.

Initial laboratory studies showed pancytopenia with neutropenia (absolute neutrophil count [ANC], $150 / \mu \mathrm{L}$; reference, $>1,500 / \mu \mathrm{L}$ ), hyponatremia (131 mmol/L), and increased prothrombin time (12.9 seconds) and partial thromboplastin time (41.1 seconds). C-reactive protein $(40.3 \mathrm{mg} / \mathrm{L})$ and erythrocyte sedimentation rate $(59 \mathrm{~mm} / \mathrm{h})$ were also elevated. Additional routine laboratory test results are shown in Table 1. Screening for infection yielded no evidence of cytomegalovirus infection, or other causes of atypical pneumonia. Cultures of the blood and sputum were negative, but urine culture yielded 1,000 colony-forming units/mL of Gram-positive cocci. On chest X-ray, multiple nodular consolidations were observed in the left upper lung, and a chest computed tomography (CT) scan further demonstrated multiple nodular consolidations in the right upper lobe with a small amount of left pleural effusion and pericardial effusion. No enlarged lymph nodes were identified, and the enlarged spleen measured $21.4 \mathrm{~cm}$.

Initial medications upon admission included aspirin, nicorandil, trimetazidine, bisoprolol, and levothyroxine for the treatment of the underlying conditions. Moreover, total parenteral nutrition was initiated to compensate for the patient's nutritional deficit. Antibiotic therapy was commenced with trimethoprim/sulfamethoxazole, meropen$\mathrm{em}$, and amphotericin B, as was used in the previous clinic. However, as the chest CT scan indicated minimal possibility of active Pneumocystis pneumonia, and polymerase chain reaction for Pneumocystis jirovecii was also negative, amphotericin B was administered as the sole antifungal agent. Additionally, prophylactic antibacterial agents were reduced to cefepime, since the fever had subsided by the time the patient was transferred to Severance Hospital.

Consultations from the departments of hematology, rheumatology, and infection were sought, and the differential diagnosis focused on the evaluation of splenomegaly, pancytopenia, and neutropenia. Peripheral blood smear revealed normochromic, normocytic anemia with a reticulocyte percentage of $0.65 \%$. Screening test results for iron deficiency anemia indicated anemia of chronic disease, with elevated

Table 1. Initial laboratory investigations performed on day 1 of admission (7/16/2019)

\begin{tabular}{|c|c|}
\hline Test & Results \\
\hline Complete blood count & Total WBC $540 / \mu \mathrm{L}$ (neutrophil $28 \%$, lymphocyte $51.6 \%$, monocyte $13.1 \%$ ), hemoglobin $9.9 \mathrm{~g} / \mathrm{dL}$, platelet count $37 \times 10^{3} / \mu \mathrm{L}$ \\
\hline Electrolytes & Na $131 \mathrm{mmol} / \mathrm{L}, \mathrm{K} 4.1 \mathrm{mmol} / \mathrm{L}, \mathrm{Cl} 101 \mathrm{mmol} / \mathrm{L}, \mathrm{Ca} 7.5 \mathrm{mg} / \mathrm{dL}, \mathrm{P} 2.5 \mathrm{mg} / \mathrm{dL}$ \\
\hline Liver function test & $\begin{array}{l}\text { Albumin } 2.7 \mathrm{~g} / \mathrm{dL} \text {, alkaline phosphatase } 147 \mathrm{IU} / \mathrm{L} \text {, aspartate aminotransferase } 30 \mathrm{IU} / \mathrm{L} \text {, alanine aminotransferase } 19 \mathrm{IU} / \mathrm{L} \text {, } \\
\text { gamma-glutamyltransferase } 75 \mathrm{IU} / \mathrm{L} \text {, bilirubin } 0.8 \mathrm{mg} / \mathrm{dL}\end{array}$ \\
\hline Renal function test & Blood urea nitrogen $14.8 \mathrm{mg} / \mathrm{dL}$, creatinine $0.8 \mathrm{mg} / \mathrm{dL}$ \\
\hline Urine analysis with microscopy & Color: yellow, turbidity: clear, pH 7.0, protein: trace, red blood cells 0-2/HPF, WBC 0-2/HPF \\
\hline Blood coagulation test & Prothrombin time $12.9 \mathrm{~s}$, activated partial thromboplastin time $41.1 \mathrm{~s}$ \\
\hline Cardiac markers & CK 34 IU/L, CK-muscle/brain $1.5 \mathrm{ng} / \mathrm{mL}$, troponin-T $26 \mathrm{pg} / \mathrm{mL}$, N-terminal prohormone of brain natriuretic peptide 1,284 pg/mL \\
\hline Inflammatory markers & C-reactive protein $40.3 \mathrm{mg} / \mathrm{L}$, erythrocyte sedimentation rate $59 \mathrm{~mm} / \mathrm{h}$ \\
\hline
\end{tabular}

WBC, white blood cells; HPF, high power field; CK, creatine kinase. 
ferritin $(3,308.5 \mathrm{ng} / \mathrm{mL})$ and low transferrin $(98 \mathrm{mg} / \mathrm{dL})$ along with normal serum iron level $(79 \mu \mathrm{g} / \mathrm{dL})$. In addition, there was no deficiency of B12 and folate. Bone marrow biopsy revealed an average cellularity of $60 \%$, with no signs of bone marrow failure. The detailed results can be found in Table 2, and follow-up results for complete blood counts are listed in Table 3.

Prior bone marrow biopsies had similarly failed to indicate a diagnosis, which made primary hematologic disease unlikely. Subsequently, investigations were performed for potential autoimmune causes, and these yielded positive anti-nuclear antibodies (ANA) with a titer of 320:1 and rheumatoid factor (RF) levels of $23 \mathrm{IU} / \mathrm{mL}$. After a rheumatology consultation, further studies were performed revealing weakly-positive anti-DNA (19 IU/mL) and anti-cardiolipin immunoglobulin M antibodies (11 MPL-U/mL). Anti-beta2-glycoprotein 1 antibodies and lupus anticoagulant were both negative. Serum C3 was low (36.7 mg/dL), but C4 was normal (11.98 mg/dL). Furthermore, when the patient was evaluated for lupoid manifestations, an oral ulcer was found, but no other autoimmune features were seen, including skin rash, arthritis, muscular weakness, uveitis, and Reynaud phenomenon. Cystatin C was elevated (1.53 mg/L), and cystatin C-based estimated glomerular filtration rate (GFR) was $42.6 \mathrm{~mL} / \mathrm{min} / 1.73 \mathrm{~m}^{2}$. Although the creatinine level was normal $(0.82 \mathrm{mg} / \mathrm{dL})$, its diagnostic value was limited due to the low BMI of the patient. Considering the ongoing infection, the presence of renal failure was deemed indeterminate. Follow-up investigations showed equivocal levels of anti-DNA antibodies (10 IU/mL), therefore, the elevation of rheumatologic antibodies was tentatively considered to be a by-product of infection upon further consultation.
With other possible causes of pancytopenia ruled out, splenomegaly itself was suspected to be the cause of the pancytopenia. During the hospitalization, the pancytopenia progressively worsened, and by day 11 of hospitalization, total white cell count reached $540 / \mu \mathrm{L}$ (neutrophil $35.9 \%$ ), hemoglobin $6.8 \mathrm{~g} / \mathrm{dL}$, and platelet count $57 \times 10^{3} / \mu \mathrm{L}$. Moreover, onset of sepsis was suspected, with body temperature of $37.0^{\circ} \mathrm{C}$, blood pressure of 86/52 mm Hg, and pulse rate of 84 . After an infusion of 1.5 L of fluid did not raise the blood pressure to baseline levels, filtered red blood cells were transfused, along with norepinephrine at a rate of 4 $\mathrm{mL} / \mathrm{h}$. Given the patient's indeterminate diagnosis and deteriorating general condition, splenectomy was considered as a curative treatment option. However, considering the poor condition of the patient, surgical splenectomy was deemed as hazardous, and partial splenic embolization was considered as an alternative. During the course of hospitalization, numerous consultations were sought but due to the failure to establish a definite diagnosis, no specific department could agree to actively transfer the patient from our department. Partial splenic embolization was undertaken on day 17 of hospitalization, using iodixanol as a contrast agent, and ultrasound guidance for arterial access. Vascular access point was right common femoral artery, and after performing celiac angiography and splenic angiography, the lower portion (70\%) of the spleen was embolized with gelfoam. Intra-procedural images are shown in Figure 1.

The day after embolization, there were no serious complications and a general increase in blood counts was observed; white blood cell count rose to $820 / \mu \mathrm{L}$, hemoglobin to $10.0 \mathrm{~g} / \mathrm{dL}$, and platelet count was $45 \times 10^{3} / \mu \mathrm{L}$. However, 2 days after the procedure, at $11 \mathrm{PM}$, the patient's temperature rose to $38.5^{\circ} \mathrm{C}$, followed by a rapid drop to $35.7^{\circ} \mathrm{C}$ in an

Table 2. Screening investigations for hematologic disease

\begin{tabular}{|c|c|c|}
\hline Test & Date & Results \\
\hline Peripheral blood smear & $7 / 17 / 2019$ & Normocytic normochromic anemia with anisocytosis; marked leukopenia; moderate thrombocytopenia \\
\hline Iron deficiency evaluation & $7 / 17 / 2019$ & $\begin{array}{l}\text { Serum iron } 79 \mu \mathrm{g} / \mathrm{dL} \text {; total iron binding capacity } 140 \mu \mathrm{g} / \mathrm{dL} \text {; transferrin saturation } 56 \% \text {; ferritin 3,308.5 } \mathrm{ng} / \mathrm{mL} \text {; } \\
\text { transferrin } 98 \mathrm{mg} / \mathrm{dL}\end{array}$ \\
\hline Vitamin B12 \& folate & 7/17/2019 & Vitamin B12 12,334 pg/mL; folate $8.98 \mathrm{ng} / \mathrm{mL}$ \\
\hline Bone marrow biopsy & $7 / 19 / 2019$ & $\begin{array}{l}\text { Average cellularity 60\%; normal myeloid/erythroid ratio; full maturation of erythroid and myeloid series; shift to left of } \\
\text { myeloid series; adequate number of megakaryocytes; interstitial hemosiderin deposition and infiltration of numerous } \\
\text { CD3+ T-cells and some CD138+ plasma cells, favor reactive; reticulin stain: grade } 0 \text { myelofibrosis (normal) }\end{array}$ \\
\hline
\end{tabular}

Table 3. Complete blood count and C-reactive protein on follow-up (7/16/2019-8/4/2019)

\begin{tabular}{|c|c|c|c|c|}
\hline Date & White blood cell count $\left(10^{3} / \mu \mathrm{L}\right)$ & Hemoglobin (g/dL) & Platelet count $\left(10^{3} / \mu \mathrm{L}\right)$ & C-reactive protein (mg/L) \\
\hline Reference & (Range, 4.0-10.8) & (Range, 13.0-17.4) & (Range, 150.0-400.0) & (Range, 0-8) \\
\hline $7 / 16$ & 0.54 & 9.9 & 37 & 40.3 \\
\hline $7 / 19$ & 0.52 & 9.5 & 22 & 29.0 \\
\hline $7 / 20$ & 0.51 & 8.5 & 23 & 32.4 \\
\hline $7 / 21$ & 0.68 & 9.5 & 23 & 40.9 \\
\hline $7 / 22$ & 0.75 & 9.1 & 35 & 53.7 \\
\hline $7 / 23$ & 0.67 & 8 & 41 & 79.0 \\
\hline $7 / 25$ & 0.73 & 8.4 & 56 & 79.7 \\
\hline $7 / 26$ & 0.59 & 8.4 & 54 & - \\
\hline $7 / 27$ & 0.73 & 8.6 & 52 & 66.1 \\
\hline $7 / 28$ & 0.75 & 7.9 & 62 & - \\
\hline
\end{tabular}



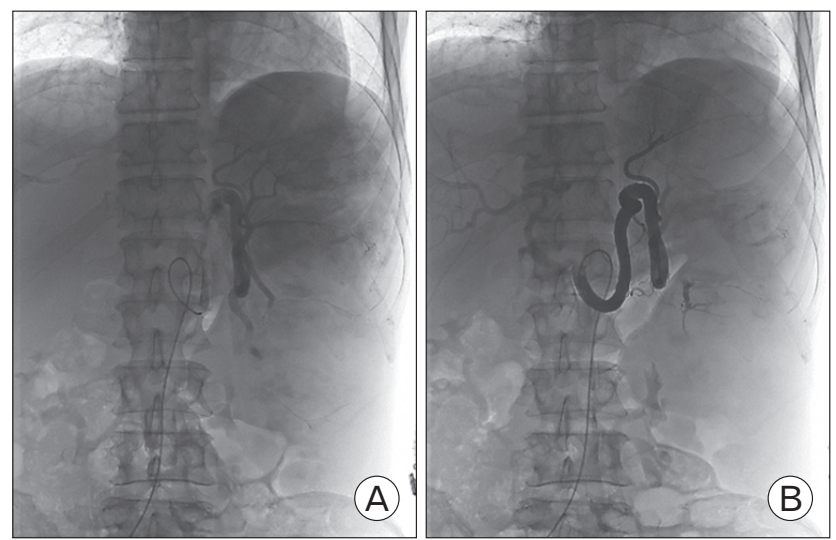

Figure 1. (A, B) Splenic angiography images, taken on August 1, 2019, before (A) and after partial splenic embolization (B).

hour accompanied by a decrease in blood pressure to $84 / 56 \mathrm{~mm} \mathrm{Hg}$ and a pulse rate increase to 148 per minute. Septic shock was suspected; therefore, norepinephrine and fluids were infused and CT scans of the chest and abdomen were undertaken. Notably, there was no pulmonary embolism or bowel perforation, and the abdominal CT image is shown in Figure 2. After 2 hours of resuscitation, increased rales were heard in the lungs, and oxygen saturation began to fall, eventually reaching 55\%. Consequently, mechanical ventilation was initiated. However, given the grim prognosis, the legal guardians of the patient refused to give consent for admission to the intensive care unit for closer monitoring and treatment. The patient began to show signs of cardiac arrest at $5 \mathrm{AM}$, when cardiopulmonary resuscitation (CPR) was commenced. Nevertheless, spontaneous circulation could not be established and the patient died 60 minutes into CPR at 6 AM.

Written informed consent for publication of this image was obtained from the patient.

\section{DISCUSSION}

This case report describes a case of pancytopenia with splenomegaly in an elderly man. Specifically, pancytopenia and accompanying neutropenia (initial ANC $88 / \mu \mathrm{L}$ ) were the major issues, and a history of multiple, long-term admissions was severely jeopardizing the patient's quality of life. No definite diagnosis could be made in spite of a range of rheumatologic and hematologic evaluations conducted in our hospital and in previous hospitals. All investigations yielded similar results and did not meet any specific diagnostic criteria. This suggested a diagnostic 'gray zone,' where the patient's general condition clearly indicated severe pathologic changes, but no definite diagnosis could be assigned; thus, this case could be described as a case of ICUS. ICUS is defined as an unexplained persistent cytopenia which fails to be diagnosed as myelodysplastic syndrome (MDS) by the accepted criteria. Cytopenia must be substantial and observed for at least 6 months, and upon diagnosis, a thorough hematologic follow-up, similar to that in low-risk MDS is warranted due to its possible progression to MDS or

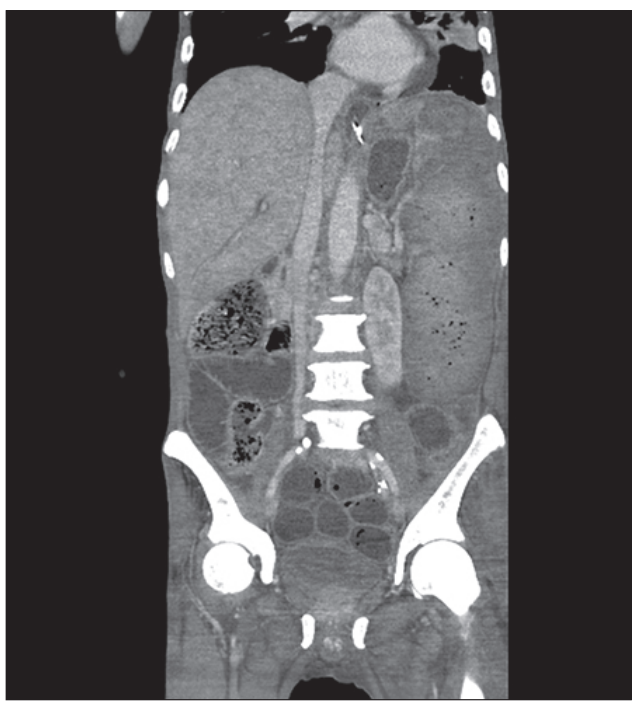

Figure 2. Abdominal computed tomography performed on August 4, 2019, at the first signs of septic shock. The image shows marked infarction of spleen with air bubbles and diffuse dilatation of small bowel loops without a transition point.

another myeloid disorder over time. ${ }^{3)}$

The differential diagnoses we considered were aplastic anemia, leukemia, myelofibrosis, and MDS among hematologic causes, and systemic lupus erythematosus (SLE) among autoimmune causes. With respect to hematologic causes, multiple bone marrow biopsies performed at the current and previous hospitals failed to reveal signs of a hypocellular marrow, ineffective hematopoiesis, or myelofibrosis. For rheumatologic causes, the presence of ANA and RF was investigated, and mildly elevated anti-dsDNA antibodies were discovered. Although anti-dsDNA antibodies measured by enzyme-linked immunosorbent assay generally have a high specificity, low or equivocal titers may be induced by conditions other than SLE. ${ }^{4)}$ Specifically, moderately positive titers indicate $72 \%$ specificity and equivocal titers indicate $65 \%$ specificity. ${ }^{5)}$ As the titers never reached strongly positive levels and rather, decreased over time, we presumed that sepsis was responsible for the transient elevation of anti-dsDNA antibodies and rheumatologic factor. As for the presence of renal dysfunction seen in lupus nephritis, both creatinine and cystatin $\mathrm{C}$ were measured to estimate the GFR, but the accuracy of creatinine-based estimated GFR was questionable due to the low BMI. ${ }^{6}$ Additionally, cystatin $\mathrm{C}$ is also known to be affected by positive $\mathrm{RF}^{\text {; }}{ }^{7}$ therefore, the exact renal function status of the patient could not be ascertained.

Splenectomy was initially considered as a curative treatment as splenic sequestration was suspected due to the massive splenomegaly. Furthermore, it has been previously reported that splenectomy may have a curative effect in such cases. ${ }^{1)}$ However, as the patient was critically ill, partial splenic embolism was performed instead to avoid the risks of surgery. ${ }^{8)}$ Unfortunately, these measures failed to prevent the eventual death of the patient from septic shock.

The role of family medicine is often described as 'providing primary care from birth to the end of life.' In the present case, we found our- 
selves in a dilemma regarding the optimal management of the patient. This case represented a diagnostic 'gray zone,' where the referral was rejected while no definitive treatment options could be decided upon even after consultations, due to the uncertain diagnosis. However, the condition was having a severely detrimental impact on the quality of life of the patient with no notable improvement over the long clinical course; therefore, the desire of the patient and his family for definitive treatment was considered before deciding the management plan. With consultations regarding medically unexplained physical symptoms becoming more common in recent times, similar dilemmas may occur in such 'gray zone' patients. Currently, although all subspecialties put significant emphasis on disease management, less attention may be paid to the desires of the patients and their families. Family medicine may fill in the gaps here. As providers of comprehensive care, we can optimally incorporate the personal values of the patients and their families into the medical decisions, and thus help in deciding the best course of action in similar situations. We hope that our experience in this case may give some insight into the management of future patients in the 'gray zone.'

\section{CONFLICT OF INTEREST}

No potential conflict of interest relevant to this article was reported.

\section{ORCID}

Jaehee Cho: https://orcid.org/0000-0001-7012-1623

Youhyun Song: https://orcid.org/0000-0001-5621-2107

Jiyeon Lee: https://orcid.org/0000-0003-2213-5558

Daeun Lee: https://orcid.org/0000-0003-3731-548X
Yunsun Go: https://orcid.org/0000-0001-9900-7226

Hee Cheol Kang: https://orcid.org/0000-0002-0309-7448

\section{REFERENCES}

1. Weinzierl EP, Arber DA. The differential diagnosis and bone marrow evaluation of new-onset pancytopenia. Am J Clin Pathol 2013;139:929.

2. Bae MH, Cho YU, Kim B, Jang S, Park CJ, Chang YH, et al. Pancytopenia or Bicytopenia in a Korean Tertiary Care Center; Etiological profile based on bone marrow examination and suggestion for diagnostic approach. Blood 2015;126:5610.

3. Valent P, Bain BJ, Bennett JM, Wimazal F, Sperr WR, Mufti G, et al. Idiopathic cytopenia of undetermined significance (ICUS) and idiopathic dysplasia of uncertain significance (IDUS), and their distinction from low risk MDS. Leuk Res 2012;36:1-5.

4. Kavanaugh AF, Solomon DH; American College of Rheumatology Ad Hoc Committee on Immunologic Testing Guidelines. Guidelines for immunologic laboratory testing in the rheumatic diseases: anti-DNA antibody tests. Arthritis Rheum 2002;47:546-55.

5. Attar SM, Koshak EA. Medical conditions associated with a positive anti-double-stranded deoxyribonucleic acid. Saudi Med J 2010;31: 781-7.

6. Shlipak MG, Mattes MD, Peralta CA. Update on cystatin C: incorporation into clinical practice. Am J Kidney Dis 2013;62:595-603.

7. Chew JS, Saleem M, Florkowski CM, George PM. Cystatin C: a paradigm of evidence based laboratory medicine. Clin Biochem Rev 2008;29:47-62.

8. Mousa A, Armbruster J, Adongay J, AbuRahma AF. Splenic artery embolization as a treatment option for chronic pancytopenia secondary to hypersplenism: a case report and review of literature. Vasc Endovascular Surg 2012;46:501-3. 